\title{
Molecular Evidence of Interhuman Transmission of Pneumocystis Pneumonia among Renal Transplant Recipients Hospitalized with HIV-Infected Patients
}

\author{
Meja Rabodonirina, ${ }^{\star 1}$ Philippe Vanhems, $† \S$ Sandrine Couray-Targe, $\ddagger$ René-Pierre Gillibert, $\dagger$ \\ Christell Ganne, $\ddagger$ Nathalie Nizard, $\uparrow$ Cyrille Colin, $\neq$ Jacques Fabry, $\uparrow \S$ Jean-Louis Touraine, $\S$

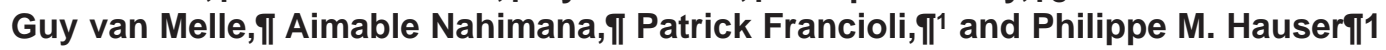

Ten Pneumocystis jirovecii pneumonia (PCP) cases were diagnosed in renal transplant recipients (RTRs) during a 3-year period. Nosocomial transmission from HIVpositive patients with PCP was suspected because these patients shared the same hospital building, were not isolated, and were receiving suboptimal or no anti-PCP prophylaxis. P. jirovecii organisms were typed with the multitarget polymerase chain reaction-single-strand conformation polymorphism method. Among the 45 patients with PCP hospitalized during the 3-year period, 8 RTRs and $6 \mathrm{HIV}$-infected patients may have encountered at least 1 patient with active PCP within the 3 months before the diagnosis of their own PCP episode. In six instances (five RTRs, one HIV-infected patient), the patients harbored the same $P$. jirovecii molecular type as that found in the encountered PCP patients. The data suggest that part of the PCP cases observed in this building, particularly those observed in RTRs, were related to nosocomial interhuman transmission.

Deumocystis jirovecii pneumonia (PCP) is a severe 1 opportunistic infection in immunocompromised patients $(1,2)$. It remains a major problem in some HIVinfected persons who are not receiving or not responding to highly active antiretroviral triple therapy and among those who are unaware of their HIV status. PCP is also of

*Hôpital de la Croix-Rousse, Lyon, France; †Université Claude Bernard and INSERM U271, Lyon, France; łHospices Civils de Lyon, Lyon, France; §Hôpital Edouard-Herriot, Lyon, France; and ๆCentre Hospitalier Universitaire Vaudois, Lausanne, Switzerland clinical importance in immunosuppressed patients, e.g., transplant recipients and those receiving chemotherapy for malignant diseases, who are not infected with HIV. Host specificity suggests that the reservoir of $P$. jirovecii is limited to humans. Primary infection in infants $(3,4)$, as well as asymptomatic carriage by immunosuppressed persons (5-8), may serve as infectious reservoirs or sources in the community. Reactivation of a past infection was a postulate mechanism of infection in immunosuppressed patients, but de novo infection in recurrent episodes of the disease (9) has suggested that infection or reinfection from exogenous sources may occur. Horizontal airborne transmission has been demonstrated in several animal models (10-12).

Transmission of $P$. jirovecii from patients with active PCP to susceptible persons has been suspected in numerous descriptions of nosocomial clusters of PCP cases (13-17). Although a common environmental source of the infection was difficult to exclude, many patients in the clusters had contact with each other, which suggests that they may have transmitted $P$. jirovecii to one another. The early reports of PCP epidemics among malnourished children in orphanages and hospitals in the 1950s were also compatible with interhuman transmission of $P$. jirovecii (18). The strongest suspicion of transmission was provided by a case-control study performed for a cluster of five PCP cases in transplant recipients (13). This analysis

${ }^{1}$ M. Rabodonirina, P. Francioli, and P.M. Hauser contributed equally to the work. 
showed that the patients had more encounters than matched controls at the outpatient facility with HIV patients who had or subsequently developed PCP. However, in these studies, transmission of $P$. jirovecii could not be assessed at the molecular level because no molecular typing method for $P$. jirovecii existed. Such methods were developed in the 1990s, and new clusters were analyzed. However, the few published anecdotal analyses often reported different genotypes within the clusters (19-21). Thus, interhuman transmission of $P$. jirovecii from PCP cases is still an open issue.

The latest guidelines developed by the U.S. Public Health Service and the Infectious Diseases Society of America for preventing opportunistic infections in persons infected with HIV state that although some authorities recommend that persons who are at risk for $P$. jirovecii pneumonia not share a hospital room with a patient who has PCP, data are insufficient to support this recommendation as standard practice $(22,23)$.

In our molecular epidemiologic study, we investigated the possibility of $P$. jirovecii transmission between persons during a 3-year period in a hospital building that simultaneously hosted HIV patients (with and without PCP) and renal transplant recipients (RTR) (often during rejection episodes), and in which a cluster of PCP was observed.

\section{Material and Methods}

\section{Hospital Setting}

Edouard-Herriot Hospital is a 1,200-bed healthcare facility in Lyon, France, and is made up of several buildings. One of these buildings (building A, 80 beds) accommodates one hospital ward, an intensive care unit, an outpatient clinic, and a radiodiagnostic facility, which are mostly devoted to renal transplant medicine and clinical immunology, including HIV medicine. Another building (building B) hosts only patients with hematologic malignancies and is located $100 \mathrm{~m}$ away from building $\mathrm{A}$.

\section{Data Collection}

Our investigation included the 39 patients with PCP who were hospitalized in building $\mathrm{A}$ and whose bronchoalveolar lavage (BAL) specimen was available for molecular typing. These patients were chosen because interhuman transmission of $P$. jirovecii was suspected in this building. The database of the Department of Medical Information of the University Hospitals of Lyon was used to identify the demographic and clinical characteristics of the patients. Relevant data (prophylaxis regimen, hospitalization periods, dates of outpatient visits, immunosuppressive regimen) were also extracted from the medical charts of patients by using a questionnaire and log books of the outpatient clinic and radiodiagnostic facility.

\section{Laboratory Diagnosis and Storage of Specimens}

PCP was diagnosed by using methenamine-silver nitrate (24) and Giemsa stains on BAL specimens in the parasitology laboratory of Claude-Bernard University, which has processed all specimens using the same techniques for many years. The number of BAL specimens submitted for patients seen at Edouard-Herriot Hospital has been stable over the years (1992-1998: 235, 241, 277, 254, 290, 215, 215, respectively). BAL specimens of patients with proven PCP were stored at $-20^{\circ} \mathrm{C}$.

\section{Molecular Typing}

BAL specimens were typed as described previously (25-27) with the polymerase chain reaction (PCR)-singlestrand conformation polymorphism (SSCP) method for typing $P$. carinii, now named $P$. jirovecii (28), in humans. The method consists of amplifying four variable regions of the $P$. jirovecii genome, followed by the detecting the polymorphisms with SSCP. The variable regions analyzed are the internal transcribed spacer 1 of the nuclear rDNA operon, the intron of the nuclear 26S rRNA gene, the variable region of the mitochondrial 26S rRNA gene, and the region surrounding the intron 6 of the $\beta$-tubulin gene. The different SSCP patterns observed are caused by one to four base-pair polymorphisms (26). A P. jirovecii type is defined by a combination of four alleles, which corresponds to the four genomic regions. If a specimen harbors two alleles of one or more of the four genomic regions, the patient was considered coinfected with two or more $P$. jirovecii types (25). For a given patient, each type is defined as an "isolate." Molecular typing was performed on specimens from patients in building A from 1994 to 1996, as well as on representative specimens collected during the same period in building B of the Edouard-Herriot hospital and in other university hospitals of Lyon. In addition, the dihydropteroate synthase (DHPS) genotype was determined by using PCR-SSCP as described (29). Four DHPS alleles have been described in $P$. jirovecii (30). The mutated alleles result in an amino acid change in the active site of the enzyme at position 55 (allele M1) or 57 (M2), or both polymorphisms (M3).

\section{Definitions}

In the absence of knowledge of many biologic and epidemiologic characteristics of $P$. jirovecii infection, the incubation period of the not yet symptomatic patients and the period of infectivity of patients with PCP were postulated on the basis of available human and experimental data. Described clusters of PCP $(13,14)$ suggest that the incubation period of de novo infection is 3-12 weeks. Accordingly, we assumed that a new infection with $P$. jirovecii (as opposed to reactivation) would occur 3-12 weeks before laboratory diagnosis of PCP. This finding is 
also in accordance with experiments in animals (31-33). Similarly, we considered that the risk of transmission from a P. jirovecii-infected patient to a susceptible one was likely to be highest from early symptoms to the middle of treatment. We assumed that an infected patient could transmit $P$. jirovecii from 3 weeks before to 2 weeks after the PCP diagnosis. We hypothesized that transmission was airborne and defined that a potentially infectious encounter occurred if a patient within his or her susceptible period and another patient within his or her infectious period visited the same location in building A on the same day. Transmission was considered possible if the patients encountered at least once and shared a common P. jirovecii type.

\section{PCP Prophylaxis, Isolation, and Immunosuppression}

Four HIV-infected patients and four transplant recipients at risk for PCP were receiving sulfadoxinepyrimethamine, but at a dosage lower than recommended for anti-P. jirovecii prophylaxis (25 mg pyrimethamine plus $500 \mathrm{mg}$ sulfadoxine in one tablet taken once a week or every 2 weeks versus two tablets per week [34]). In addition, four HIV-infected patients were receiving aerosolized pentamidine (300 mg every 2 weeks). The other 27 patients did not receive any anti-Pneumocystis prophylaxis. A policy for isolating patients according to their underlying disease or to the occurrence of a PCP episode did not exist. Patients were allowed to move freely in the units and shared a TV room when permitted by their general condition. All RTRs, including those who experienced PCP, received usual inductive and maintenance treatments with prednisone, azathioprine, and cyclosporine. Treating rejection included high-dose corticosteroids and, if necessary, monoclonal antibodies.

\section{Case-Case Comparison}

Case-case comparison based on molecular typing of the pathogen (35) was performed. Groups of cases infected with different $P$. jirovecii molecular types were compared to investigate differences in exposure histories.

\section{Results}

From 1994 to 1996, a total of 45 patients with 46 episodes of PCP were hospitalized in building A of the Edouard-Herriot Hospital. Their age ranged from 23 to 56 years (median 41), and most of them were male (82\%). Thirty-six episodes were observed in 35 HIV-infected patients and 10 episodes in 10 RTRs. Thirty-one HIVinfected patients were admitted because of PCP, and PCP developed in all 10 RTRs and 4 HIV-infected patients during or shortly after hospitalization. These numbers represented a substantial increase compared to previous years, particularly in RTRs in whom only one case had been diag-

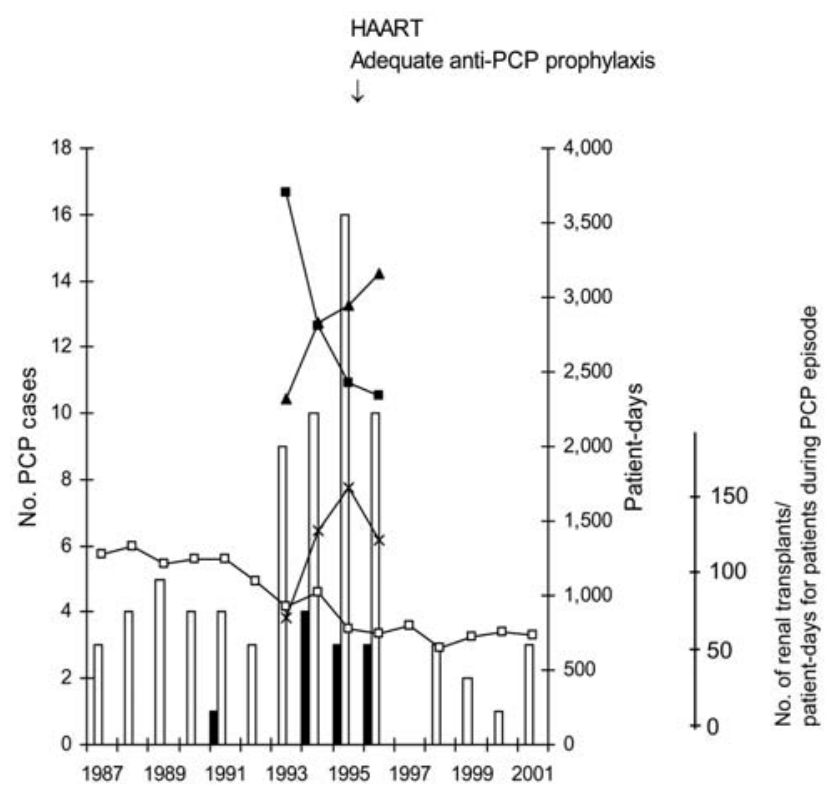

Figure 1. Pneumocystis jirovecii pneumonia (PCP) cases in HIVinfected patients (white bars) and in transplant recipients (black bars) at building A of Edouard-Herriot Hospital. Solid lines show the number of hospital patient-days for transplant recipients (filled squares), for HIV-infected patients (filled triangles), and for the patients during their PCP episode (crosses), as well as the number of renal transplantations performed (white squares). HAART, highly active antiretroviral therapy.

nosed during the 7 preceding years (Figure 1; the likelihood ratio for equality of two Poisson processes yields $\mathrm{p}=$ 0.00002; 1 case in 7 years versus 10 in 3 years). During the period from 1993 to 1996, the number of hospital patientdays for RTRs decreased by $37 \%$, whereas those of the populations of HIV-infected patients and of patients with PCP increased, respectively, by 36\% and 63\% (Figure 1). The number of admissions of HIV-infected patients in building A was 339 in 1993, 364 in 1994, 401 in 1995, and 445 in 1996. The number of admissions of RTRs was 469 in 1993, 311 in 1994, 319 in 1995, and 297 in 1996. Precise admission figures before 1993 are not available, but the number of renal transplants performed at EdouardHerriot Hospital has been decreasing from 128 in 1987 to 74 in 1996 and has been stable since then (Figure 1). The immunosuppressive regimen has not changed for RTRs from 1990 to 1997 in Edouard-Herriot Hospital.

\section{Molecular Typing $P$. jirovecii}

Thirty-nine of the 46 BAL specimens collected from 1994 to 1996 were available for typing (30 in HIV-infected patients and 9 in transplant recipients). Nineteen (49\%) specimens corresponded to an infection with a single $P$. jirovecii type, 15 (38\%) with two types, and 5 (13\%) with more than two types. A total of 19 different $P$. jirovecii types were observed. In building A, the frequency of each 
type was $2 \%-12 \%$ of the $P$. jirovecii isolates, except for type 1 which represented $39 \%$ of the isolates and was isolated in 19 patients. Type 1, the most prevalent, represented $10 \%-20 \%$ of the isolates in Switzerland and other European cities (27), as well as in building B and other hospital facilities of Lyon (Figure 2). In particular, the frequency of type 1 was significantly higher in building A than in the other hospital facilities of Lyon (19 of 45 versus 28 of 145, Fisher exact test $p=0.003$ ). Moreover, the frequency distribution of type 1 in the different categories of PCP patients hosted in building A was significantly different: it represented 31\% (12 of 39) of the isolates from the HIV-infected patients, but 70\% (7 of 10) of those from the transplant recipients (Figure 3, Fisher exact test $\mathrm{p}=0.033)$. Seven of the $12 \mathrm{HIV}$-infected patients infected with $P$. jirovecii type 1 also harbored another type (coinfection), whereas one of the seven transplant recipients had a coinfection.

\section{Encounters and Exposures between Patients with $P$. jirovecii}

From 1994 to 1996, 14 of 39 patients with PCP and available BAL specimens had prior encounters with patients with PCP (6 of the $30 \mathrm{HIV}$-infected patients and 8 of the 9 transplant recipients). A total of 118 potential encounters between patients with active PCP and patients who developed PCP 3-12 weeks after the encounter could be retrieved (Table). These 118 encounters corresponded to one or several encounters for the 14 patients. Among these 14 patients, PCP developed in 6 due to the same $P$. jirovecii type as 1 or 2 encountered PCP source patients, and $P$. jirovecii type 1 was involved in all 6 patients (5 transplant recipients, 1 HIV-infected patient). Of the 80 exposures involving $P$. jirovecii type 1 , six PCP episodes were observed compared to no episodes of 38 exposures not involving $P$. jirovecii type 1 .

Figure 4 shows the characteristics and chronologic events of the six putative nosocomial cases and their presumed source patients. It also shows the DHPS genotype. Five of the six nosocomial PCP patients harbored the M2 mutation and one the M3 mutation. Three nosocomial PCP patients harboring M2 mutation were receiving suboptimal anti-PCP prophylaxis. In all nosocomial cases, the presumed source patients had the same DHPS genotype. In some cases, an additional $P$. jirovecii genotype was recovered from the source patient, a finding compatible with coinfection with two types, one of which was not transmitted or present in a proportion sufficient to be detected in the nosocomial case. All the nosocomial PCP episodes in RTRs followed encounters which occurred when they were strongly immunosuppressed because of the treatment of a rejection episode (Figure 4).

Case-case comparison was used to compare exposure histories of two groups of patients, those harboring type 1 and those who did not. The proportion of patients who had at least one encounter during their susceptible period with a patient with active PCP harboring type 1 was higher in the first group (6/19 vs. 2/20), although not significant $(p=0.13$, Fisher exact test). The frequency of the M2 mutation in patients not receiving any sulfa prophylaxis

Table. Cases with Pneumocystis jirovecii pneumonia (PCP) and potential encounters 3 weeks to 3 months before their PCP episode with other patients with active PCP

\begin{tabular}{|c|c|c|c|c|c|c|c|}
\hline \multirow[b]{2}{*}{ Date of PCP } & \multirow[b]{2}{*}{$\begin{array}{l}\text { Underlying } \\
\text { disease }^{\mathrm{a}}\end{array}$} & \multirow[b]{2}{*}{$\begin{array}{l}\text { P. jirovecii PCR- } \\
\text { SSCP type }\end{array}$} & \multirow[b]{2}{*}{ CD4 counts $/ \mathrm{mm}^{3}$} & \multirow{2}{*}{$\begin{array}{l}\text { No. of } \\
\text { encounters with } \\
\text { patients with } \\
\text { active PCP }\end{array}$} & \multicolumn{2}{|c|}{$\begin{array}{l}\text { No. patients with } \\
\text { active PCP } \\
\text { encountered }\end{array}$} & \multirow[b]{2}{*}{$\begin{array}{c}\text { Presumptive } \\
\text { nosocomial PCP }\end{array}$} \\
\hline & & & & & Total & $\begin{array}{l}\text { With same } P \text {. } \\
\text { jirovecii type }\end{array}$ & \\
\hline $1 / 10 / 94$ & RTR & 1 and 6 & $-^{\circ}$ & 10 & 1 & 0 & No \\
\hline $1 / 11 / 94$ & RTR & 23 & - & 20 & 1 & 0 & No \\
\hline $11 / 5 / 94$ & RTR & Undetermined & - & 5 & 1 & 0 & No \\
\hline $12 / 6 / 94$ & HIV & 6 & 0 & 2 & 2 & 0 & No \\
\hline $12 / 6 / 94$ & RTR & 1 & - & 5 & 3 & 1 & Yes \\
\hline $12 / 13 / 94$ & HIV & 6 and 7 & 0 & 10 & 1 & 0 & No \\
\hline $1 / 31 / 95$ & HIV & 13 and 26 & 67 & 1 & 1 & 0 & No \\
\hline $9 / 20 / 95$ & HIV & 1 & 4 & 16 & 2 & 0 & No \\
\hline $10 / 20 / 95$ & HIV & 1 & 18 & 1 & 1 & 0 & No \\
\hline $12 / 28 / 95$ & RTR & 1 & - & 9 & 1 & 1 & Yes \\
\hline 2/22/96 & RTR & 1 & - & 14 & 3 & 2 & Yes \\
\hline $2 / 28 / 96$ & HIV & 1 & 0 & 14 & 2 & 1 & Yes \\
\hline $5 / 22 / 96$ & RTR & 1 & - & 8 & 7 & 1 & Yes \\
\hline $5 / 23 / 96$ & RTR & 1 & - & 3 & 3 & 1 & Yes \\
\hline Total & 8 RTRs, 6 HIV & & & 118 & & & 5 RTR, 1 HIV \\
\hline
\end{tabular}




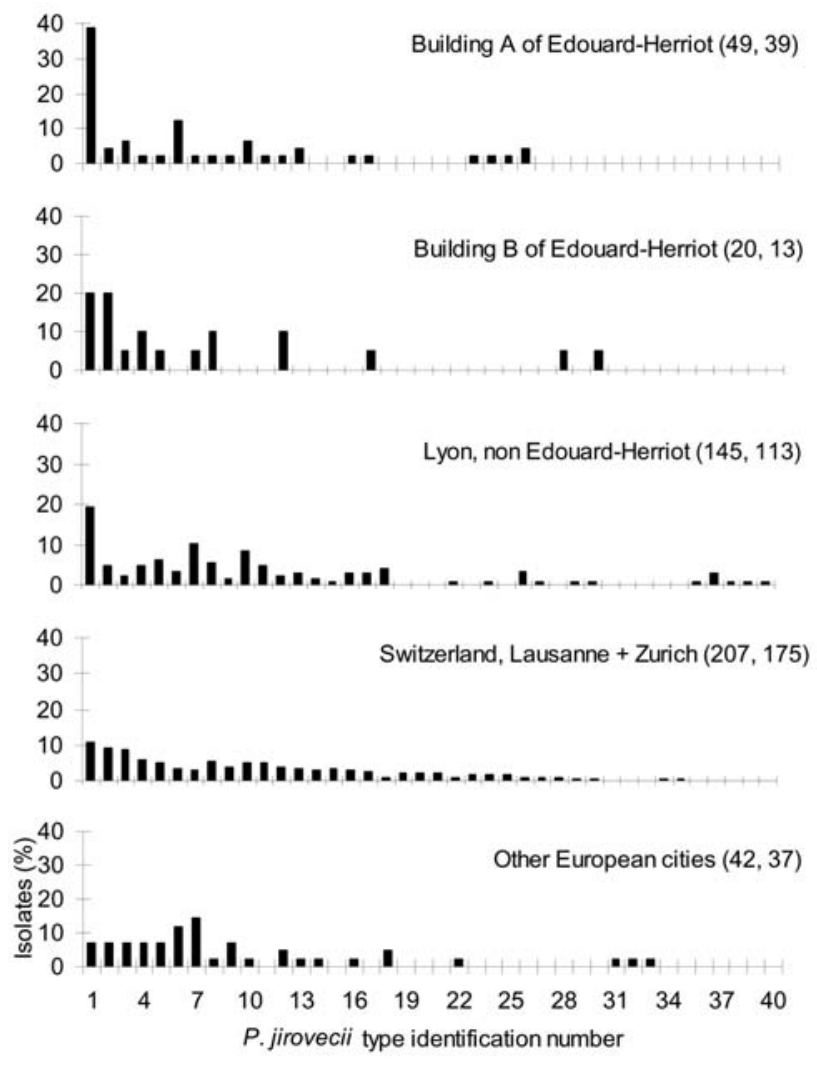

Figure 2. Frequency distribution of Pneumocystis jirovecii types observed in different cities and hospitals. Each type was considered as one isolate. The number of isolates followed by the number of specimens analyzed are indicated in the parenthesis for each geographic location. Data from Switzerland and other European cities are reproduced with permission from Hauser et al. 2001, AIDS 15(4):461-6 (27).

was significantly higher in building A than in other hospital facilities of Lyon (10/27 vs. 6/85, $\chi^{2}$ test $\left.p=0.0004\right)$.

\section{Intervention}

By mid-1996, all susceptible patients were placed on appropriate prophylaxis with co-trimoxazole (sulfamethoxazole plus trimethoprim), and HIV-infected patients had been started on highly active antiretroviral therapy. No PCP case was observed in 1997 (Figure 1), and no PCP cases were observed in transplant recipients as of December 2003.

\section{Discussion}

During a 3-year period, 10 cases of PCP in transplant recipients occurred in a building of the Edouard-Herriot Hospital in Lyon, whereas only one case was observed in the preceding 7 years. These cases could not be attributed to improved diagnosis, change of immunosuppression regimen, an increase (a decrease actually occurred) of transplant recipients hospitalized in the facility. However, the outbreak occurred concomitantly with a progressive increase in the number of HIV-infected patients with and without PCP hospitalized in the same facility. Thorough molecular and epidemiologic analyses of the PCP cases showed the following facts: 1 ) transplant recipients, often in a stage of severe immunosuppression, shared the facility with HIV-infected patients with and without active PCP; 2) both transplant recipients and HIV-infected patients were receiving no or suboptimal anti-PCP prophylaxis; 3) $P$. jirovecii type 1 represented $70 \%$ of the isolates from the transplant PCP cases, but it represented $\leq 31 \%$ of the isolates in the HIV-infected patients with PCP in Lyon and elsewhere; and 4) all the transplant recipients (and some HIV patients) in whom PCP developed had been hospitalized in the facility at some point during the 3 months preceding their PCP episodes. Moreover, the proportion of patients exposed to $P$. jirovecii type 1 during their susceptible period was higher among cases infected with type 1 than among those not harboring type 1 , although it did not reach statistical significance $(p=0.05)$. Finally, review of the medical charts indicated potential encounters between PCP cases during their susceptible period and other patients with active PCP (Table). Encounters with patients with a PCP episode involving the same $P$. jirovecii type, which may have led to transmission, were possible in 5 of the 10 transplant recipients and 1 of the 30 HIV patients. Taken together, these facts suggest that at least half of the PCP cases in transplant recipients (and possibly some in HIV patients) may be the result of a nosocomial acquisition of $P$. jirovecii.

However, alternative explanations exist that cannot be excluded. First, the transient presence of $P$. jirovecii in the

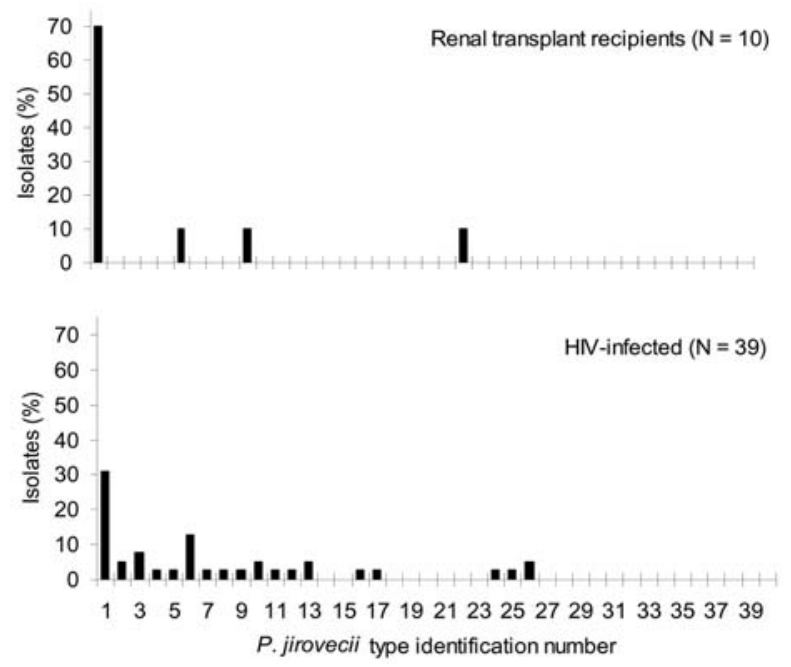

Figure 3. Frequency distribution of Pneumocystis jirovecii types observed in 30 HIV-infected patients and nine renal transplant recipients from 1994 through 1996 at building $A$ of the EdouardHerriot Hospital. 


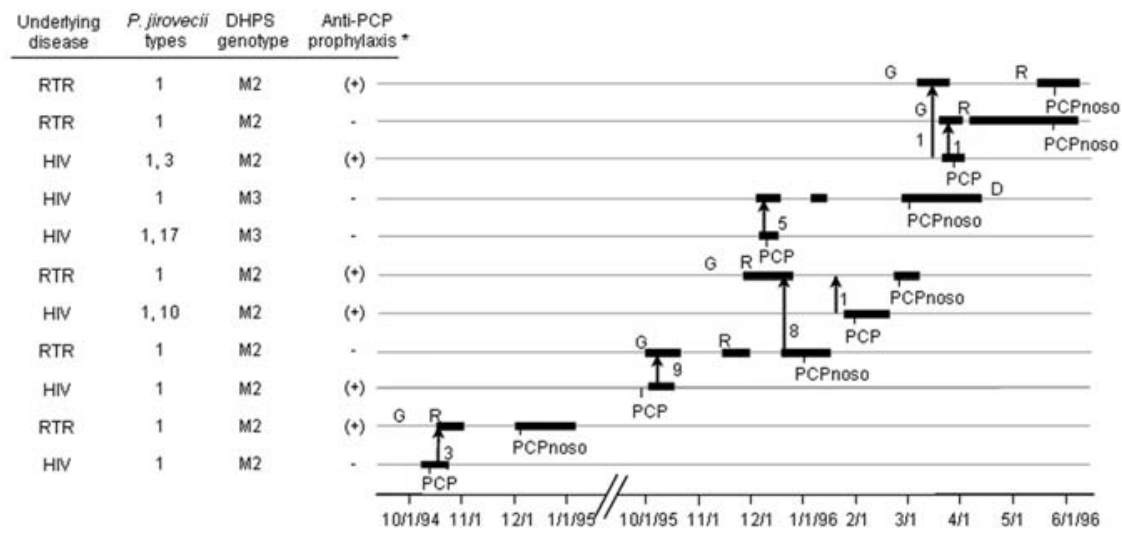

Figure 4. Potential encounters compatible with nosocomial interhuman transmission of Pneumocystis jirovecii at building A of the Edouard-Herriot Hospital (see Methods). Thicker parts of solid lines represent periods of hospitalization. Each encounter or consecutive encounters are figured by an arrow with the head indicating the direction of the presumed transmission, the number of encounters being indicated close to each arrow. *Anti-PCP prophylaxis was suboptimal. D, death. $G$, graft. $\mathrm{R}$, rejection episode. RTR, renal transplant recipient. PCPnoso, nosocomial case. air of hospital corridors has been described (36), which raises the possibility of an environmental source of $P$. jirovecii type 1 in building $\mathrm{A}$. The following facts argue against this possibility: 1) the existence of a long-lasting environmental source of $P$. jirovecii has never been established, and 2) a high prevalence of type 1 was not observed in building $\mathrm{B}$, which is located $100 \mathrm{~m}$ from building $\mathrm{A}$ and hosts patients with hematologic malignancies. Second, our study provides epidemiologic and molecular evidence that nosocomial transmission of $P$. jirovecii can occur, but whether this transmission would have occurred directly or indirectly through carriers is unclear. Indeed, carriage of $P$. jirovecii DNA has been described in the lungs of asymptomatic, immunosuppressed persons (5-8), as well as in the nose of immunocompetent relatives and healthcare workers in close contact with a PCP patient (37). Moreover, transmission by immunocompetent carriers to susceptible hosts has been demonstrated in the mouse model (38). Thus, indirect transmission through healthcare workers, physicians, or asymptomatic immunosuppressed patients cannot be ruled out.

For 14 of the 39 patients with PCP observed during the 3-year period, potential encounters with patients with active PCP during the 3 months preceding their episode had been documented (Table). However, only P. jirovecii type 1 was involved in encounters that apparently resulted in secondary cases. Part of this observation may be related to the higher prevalence of type 1 in Lyon $(\approx 20 \%)$, although this could not explain the $40 \%$ rate of type 1 in building A. Another possibility is that type 1 might be more transmissible or virulent. This finding would be consistent with the fact that this type was one of the most prevalent types also in other geographic areas (Figure 2) (27). Moreover, specific $P$. jirovecii genotypes have been associated with more severe clinical symptoms (39) or with resistance to certain drugs (40). In our study, a mutation in the active site of DHPS was present in all six presumptive nosocomial PCP cases. The mutation may have favored acquisition of type 1 rather than another type by the three patients who were receiving suboptimal prophylaxis with Fansidar (Roche, Nutley, NJ). The presence of this mutation in the nosocomial PCP cases of our study suggests that $P$. jirovecii was acquired shortly before the episode because the frequency of DHPS mutations greatly increased only in the 1990s (41). Moreover, in patients not receiving any sulfa prophylaxis, the frequency of M2 mutation was significantly higher in building A than in other hospital facilities of Lyon ( $p=0.0004)$, a fact that suggests nosocomial interhuman transmission of $P$. jirovecii.

Our study provides insight into the relative importance of nosocomial acquisition of $P$. jirovecii if infectious and susceptible patients are in close contact. Even though infected and susceptible patients were kept in unusually close proximity in this hospital, relatively few cases compatible with nosocomial interhuman transmission seem to have occurred. This finding suggests that transmission from patients with active PCP is limited, which is consistent with studies that we performed in HIV outpatient clinics that suggested infrequent cross-infections $(27,42)$, as well as with a study comparing contact histories of patients with or without PCP (43). The source remains undetermined for the infection in the five transplant recipients for whom no potentially infectious encounters were found. One possibility is that carriers of $P$. jirovecii in the hospital have played a role.

The available data and the arbitrary definitions we had to use, in light of the absence of precise scientific data on $P$. jirovecii infection, are limitations of our study. We could not demonstrate that the presumed encounters actually occurred or define the precise nature of the encounter. Also, we could not firmly exclude other potential sources of $P$. jirovecii, such as the environment or asymptomatic carriers. Nevertheless, to our knowledge, this study is the first to suggest that $P$. jirovecii may be nosocomially transmitted and acquired by severely immunosuppressed patients. Given the increased number of reports relating resistance to anti-Pneumocystis drugs, prophylaxis of 
patients at risk might not be sufficient to achieve prevention. Moreover, prophylaxis is often not satisfactory because of secondary effects. Consequently, avoiding contact between persons at risk for PCP and patients with active PCP may be warranted and should be added to prevention guidelines.

\section{Acknowledgments}

We thank Arlette Cruchon for excellent assistance, Stéphane Picot for storage of the specimens in the Laboratory of Parasitology of the Edouard-Herriot Hospital, Michèle Aymard and Danièle Thouvenot for providing some specimens from the Virology Laboratory of the Edouard-Herriot Hospital, and Giorgio Zanetti for advice on epidemiologic analyses.

This study by the Division Autonome de Médecine Préventive Hospitalière, Lausanne, was supported by grant No. 3345-6290 of the Swiss National Science foundation, and No. 00.0021 of the Swiss Federal Office for Education and Science for participation in EUROCARINII project, 5th Framework Program, European Commission (QLK2-CT-2000-01369). Aimable Nahimana was supported by a North-South fellowship from the University of Lausanne. Lyon's institutions and laboratory was supported by Research grants from Hospices Civils de Lyon, Centre de Coordination de la Lutte Contre les Infections Nosocomiales Sud-Est, Laboratoires Pfizer, Association Ensemble Contre le SIDA-Sidaction. Meja Rabodonirina is a member of the Equipe d'Accueil-3609 scientific project (EA3609) of the French Ministry of Research.

Dr. Rabodonirina is a parasitologist and a mycologist at the Hospices Civils de Lyon, as well as a lecturer at Lyon University. Her research interests include diagnosis, epidemiology, and drug resistance of opportunistic infections.

\section{References}

1. Nuesch R, Bellini C, Zimmerli W. Pneumocystis carinii pneumonia in human immunodeficiency virus (HIV)-positive and HIV-negative immunocompromised patients. Clin Infect Dis. 1999;29:1519-23.

2. Ledergerber B, Egger M, Erard V, Weber R, Hirschel B, Furrer H, et al. AIDS-related opportunistic illnesses occurring after initiation of potent antiretroviral therapy: the Swiss HIV Cohort Study. JAMA. 1999;282:2220-6.

3. Vargas SL, Hughes WT, Santolaya ME, Ulloa AV, Ponce CA, Cabrera $\mathrm{CE}$, et al. Search for primary infection by Pneumocystis carinii in a cohort of normal, healthy infants. Clin Infect Dis. 2001;32:855-61.

4. Totet A, Pautard JC, Raccurt C, Roux P, Nevez G. Genotypes at the internal transcribed spacers of the nuclear rRNA operon of Pneumocystis jiroveci in nonimmunosuppressed infants without severe pneumonia. J Clin Microbiol. 2003;41:1173-80.

5. Rabodonirina M, Cotte L, Boibieux A, Kaiser K, Mayencon M, Raffenot D, et al. Rapid detection of Pneumocystis carinii in bronchoalveolar lavage specimens from human immunodeficiency virusinfected patients: use of a simple DNA extraction procedure and nested PCR. J Clin Microbiol. 1997;35:2748-51.
6. Hauser PM, Blanc DS, Bille J, Nahimana A, Francioli P. Carriage of Pneumocystis carinii by immunosuppressed patients and molecular typing of the organisms. AIDS. 2000;14:461-3.

7. Vargas SL, Ponce CA, Sanchez CA, Ulloa AV, Bustamante R, Juarez G. Pregnancy and asymptomatic carriage of Pneumocystis jiroveci. Emerg Infect Dis. 2003;9:605-6.

8. Wakefield AE, Lindley AR, Ambrose HE, Denis CM, Miller RF. Limited asymptomatic carriage of Pneumocystis jiroveci in human immunodeficiency virus-infected patients. $\mathrm{J}$ Infect Dis. 2003;187:901-8.

9. Keely SP, Baughman RP, Smulian AG, Dohn MN, Stringer JR. Source of Pneumocystis carinii in recurrent episodes of pneumonia in AIDS patients. AIDS. 1996;10:881-8.

10. Hughes WT. Current issues in the epidemiology, transmission, and reactivation of Pneumocystis carinii. Semin Respir Infect. 1998;13:283-8.

11. Vogel P, Miller CJ, Lowenstine LL, Lackner AA. Evidence of horizontal transmission of Pneumocystis carinii pneumonia in simian immunodeficiency virus-infected rhesus macaques. J Infect Dis. 1993;168:836-43.

12. Frenkel JK, Good JT, Shultz JA. Latent Pneumocystis infection of rats, relapse, and chemotherapy. Lab Invest. 1966;15:1559-77.

13. Chave JP, David S, Wauters JP, Van Melle G, Francioli P. Transmission of Pneumocystis carinii from AIDS patients to other immunosuppressed patients: a cluster of Pneumocystis carinii pneumonia in renal transplant recipients. AIDS. 1991;5:927-32.

14. Goesch TR, Gotz G, Stellbrinck KH, Albrecht H, Weh HJ, Hossfeld DK. Possible transfer of Pneumocystis carinii between immunodeficient patients. Lancet. 1990;336:627.

15. Bensousan T, Garo B, Islam S, Bourbigot B, Cledes J, Garre M. Possible transfer of Pneumocystis carinii between kidney transplant recipients. Lancet. 1990;336:1066-7.

16. Jacobs JL, Libby DM, Winters RA, Gelmont DM, Fried ED, Hartman BJ, et al. A cluster of Pneumocystis carinii pneumonia in adults without predisposing illnesses. N Engl J Med. 1991;324:246-50.

17. Singer C, Armstrong D, Rosen PP, Shottenfeld D. Pneumocystis carinii pneumonia: a cluster of eleven cases. Ann Int Med. 1975;82:772-7.

18. Gajdusek DC. Pneumocystis carinii etiologic agent of interstitial plasma cell pneumonia of young and premature infants. Pediatrics. 1957;19:543-65.

19. Olsson M, Eriksson BM, Elvin K, Strandberg M, Wahlgren M. Genotypes of clustered cases of Pneumocystis carinii pneumonia. Scand J Infect Dis. 2001;33:285-9.

20. Latouche S, Poirot JL, Maury E, Bertrand V, Roux P. Pneumocystis carinii hominis sequencing to study hypothetical person-to-person transmission. AIDS. 1997;11:549.

21. Helweg-Larsen J, Tsolaki AG, Miller RF, Lundgren B, Wakefield AE. Clusters of Pneumocystis carinii pneumonia: analysis of person-toperson transmission by genotyping. QJM. 1998;91:813-20.

22. 2001 USPHS/IDSA guidelines for the prevention of opportunistic infections in persons infected with human immunodeficiency virus. HIV Clin Trials. 2001;2:493-554.

23. Masur H, Kaplan JE, Holmes KK. Guidelines for preventing opportunistic infections among HIV-infected persons-2002. Recommendations of the U.S. Public Health Service and the Infectious Diseases Society of America. Ann Intern Med. 2002;137:435-78.

24. Musto L, Flanigan M, Elbadawi A. Ten-minute silver stain for Pneumocystis carinii and fungi in tissue sections. Arch Pathol Lab Med. 1982;106:292-4.

25. Nahimana A, Blanc DS, Francioli P, Bille J, Hauser PM. Typing of Pneumocystis carinii f. sp. hominis by PCR-SSCP to indicate high frequency of co-infections. J Med Microbiol. 2000; 49:753-8. 
26. Hauser PM, Francioli P, Bille J, Telenti A, Blanc DS. Typing of Pneumocystis carinii f. sp. hominis by single-strand conformation polymorphism of four genomic regions. J Clin Microbiol. 1997;35:3086-91.

27. Hauser PM, Blanc DS, Sudre P, Senggen Manoloff E, Nahimana A, Bille J, et al.Genetic diversity of Pneumocystis carinii in HIV-positive and -negative patients as revealed by PCR-SSCP typing. AIDS. 2001;15:461-6.

28. Stringer JR, Beard CB, Miller RF, Wakefield AE. A new name (Pneumocystis jiroveci) for Pneumocystis from humans. Emerg Infect Dis. 2002;8:891-6.

29. Nahimana A, Rabodonirina M, Zanetti G, Meneau I, Francioli P, Bille $\mathrm{J}$, et al. Association between a specific Pneumocystis jiroveci dihydropteroate synthase mutation and failure of pyrimethamine/sulfadoxine prophylaxis in HIV-positive and -negative patients. J Infect Dis. 2003;188:1017-23.

30. Armstrong W, Meshnick S, Kazanjian P. Pneumocystis carinii mutations associated with sulfa and sulfone prophylaxis failures in immunocompromised patients. Microbes Infect. 2002;2:61-7.

31. Boylan CJ, Current WL. Improved rat model of Pneumocystis carinii pneumonia: induced laboratory infections in Pneumocystis-free animals. Infect Immun. 1992;60:1589-97.

32. Powles MA, McFadden DC, Pittarelli LA, Schmatz DM. Mouse model for Pneumocystis carinii pneumonia that uses natural transmission to initiate infection. Infect Immun. 1992;60:1397-400.

33. Board KF, Patil S, Lebedeva I, Capuano S 3rd, Trichel AM, MurpheyCorb M, et al. Experimental Pneumocystis carinii pneumonia in simian immunodeficiency virus-infected rhesus macaques. J Infect Dis. 2003;187:576-88.

34. Schurmann D, Bergmann F, Albrecht H, Padberg J, Grunewald T, Behnsch M, et al. Twice-weekly pyrimethamine-sulfadoxine effectively prevents Pneumocystis carinii pneumonia relapse and toxoplasmic encephalitis in patients with AIDS. J Infect. 2001;42:8-15.

35. McCarthy N, Giesecke J. Case-case comparisons to study causation of common infectious diseases. Int J Epidemiol. 1999;28:764-8.
36. Bartlett MS, Vermund SH, Jacobs R, Durant PJ, Shaw MM, Smith JW, Tang X, et al. Detection of Pneumocystis carinii DNA in air samples: likely environmental risk to susceptible persons. J Clin Microbiol. 1997;35:2511-3.

37. Vargas SL, Ponce CA, Gigliotti F, Ulloa AV, Prieto S, Munoz MP, et al. Transmission of Pneumocystis carinii DNA from a patient with $P$. carinii pneumonia to immunocompetent contact health care workers. J Clin Microbiol. 2000;38:1536-8.

38. Dumoulin A, Mazars E, Seguy N, Gargallo-Viola D, Vargas S, Cailliez JC, et al. Transmission of Pneumocystis carinii disease from immunocompetent contacts of infected hosts to susceptible hosts. Eur J Clin Microbiol Infect Dis. 2000;19:671-8.

39. Miller RF, Wakefield AE. Pneumocystis carinii genotypes and severity of pneumonia. Lancet. 1999;353:2039-40.

40. Hauser PM, Sudre P, Nahimana A, Francioli P, Study group. Prophylaxis failure is associated with a specific Pneumocystis carinii genotype. Clin Infect Dis. 2001;33:1080-2.

41. Helweg-Larsen J, Benfield TL, Eugen-Olsen J, Lundgren JD, Lundgren B. Effects of mutations in Pneumocystis carinii dihydropteroate synthase gene on outcome of AIDS-associated $P$. carinii pneumonia. Lancet. 1999;354:1347-51.

42. Senggen Manoloff E, Francioli P, Taffé P, Van Melle G, Bille J, Hauser PM. Risk for Pneumocystis carinii transmission among patients with pneumonia: a molecular epidemiology study. Emerg Infect Dis. 2003;9:132-4.

43. Wohl AR, Simon P, Hu YW, Duchin JS. The role of person-to-person transmission in an epidemiologic study of Pneumocystis carinii pneumonia. AIDS. 2002;16:1821-5.

Address for correspondence: P.M. Hauser, Institut de Microbiologie, Centre Hospitalier Universitaire Vaudois, 1011 Lausanne, Switzerland; fax: +41-21-314-40-60; email: Philippe.Hauser@chuv.hospvd.ch

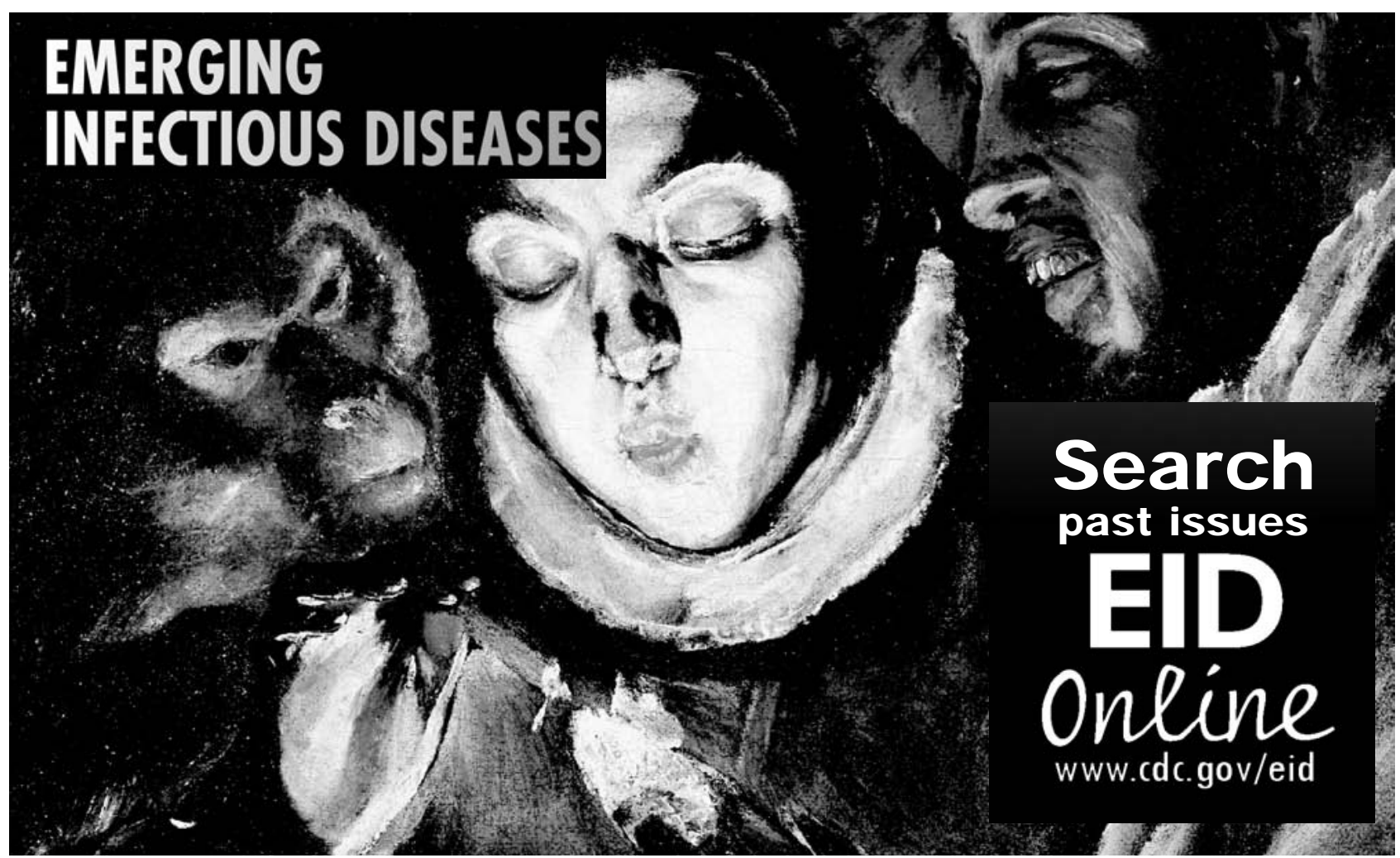

